\title{
Respek et autres mots-clés du Port-Vila urbain
}

Respek and Other Urban Vila Keywords

\section{Lamont Lindstrom}

Traducteur : Alexis Black et Christine Jourdan

\section{(2) OpenEdition Journals}

Édition électronique

URL : http://journals.openedition.org/jso/7676

DOI : $10.4000 /$ jso.7676

ISSN : $1760-7256$

\section{Éditeur}

Société des océanistes

Édition imprimée

Date de publication : 15 décembre 2017

Pagination : 23-36

ISSN : 0300-953x

Référence électronique

Lamont Lindstrom, "Respek et autres mots-clés du Port-Vila urbain », Journal de la Société des Océanistes [En ligne], 144-145 | 2017, mis en ligne le 15 décembre 2019, consulté le 15 mars 2021. URL : http://journals.openedition.org/jso/7676 ; DOI : https://doi.org/10.4000/jso.7676

\section{cc) (†)}

Journal de la société des océanistes est mis à disposition selon les termes de la Licence Creative Commons Attribution - Pas d'Utilisation Commerciale - Pas de Modification 4.0 International. 


\section{Respek et autres mots-clés du Port-Vila urbain}

par

Lamont LINDSTROM ${ }^{*}$

\section{RÉSUMÉ}

Dans leurs histoires de migration urbaine, les habitants de Tanna qui vivent dans les quartiers informels de Port-Vila emploient souvent un certain nombre de mots-clés pour décrire la vie en ville. J'utilise la méthode d'analyse culturelle dite par "mots-clés" afin d'aborder la perception insulaire de l'expérience urbaine. Respek (le respect) est un terme qui revient très fréquemment dans les entretiens que j'ai enregistrés. Ailleurs dans le monde, ce mot, souvent lié à des manouvres politiques basées sur l'ethnicité et sur le genre, a contribué de façon importante à la prédominance de ce terme. Les migrants de Tanna déplorent l'absence de respect mais l'évoquent constamment pour expliquer les conflits et les déceptions. Je mintéresse aussi aux autres mots-clés communs en bislama qui apparaissent dans les discussions sur la réalité urbaine: sikiuriti (la sécurité), mobael (à la fois le téléphone et les forces militaires du Vanuatu), noes (le bruit), jalus (la jalousie) et fri (gratuité, liberté). Ces mots-clés révèlent des perspectives instructives sur l'urbanisation mélanésienne émergeante.

MoTS-CLÉs : migrants urbains, quartiers résidentiels urbains, culture urbaine, Port-Vila, Tanna

\begin{abstract}
Telling urban migration stories, Tanna island residents of Port Vila's settlements commonly use a number of keywords to describe life in town. I follow the "keyword" method of cultural analysis to approach island appreciation of urban experience. In recorded interviews, respek (respect) was one notably frequent term. Sharpening ethnic and gender identity politicking nearly everywhere has significantly boosted the term's prominence, including in socially complex postcolonial Melanesian towns. Tanna migrants bemoan respect's absence but they evoke it constantly to explain conflict and disappointment. I also consider other common urban Bislama keywords that circulate in talk about urban reality including sekiuriti (security), mobael (both telephones and Vanuatu's military force), noes (noise), jalus (jealousy), and fri (free, freedom). Keywords unlock instructive views of emergent Melanesian urbanism.
\end{abstract}

KeYwords: urban migrants, urban settlements, urban culture, Port Vila, Tanna
Certains mots sont des clés qui ouvrent une conscience plus aiguë des formations culturelles et sociales élémentaires, du moins c'est ce que propose l'intellectuel britannique Raymond Williams dans son compte rendu historique des notions changeantes de culture et de société paru en 1958 (Culture and Society). Plus tard, en 1976, Williams publia un recueil de centdix mots-clés de ce genre, allant d' "esthétique " à «travail». Nous partageons, a dit Williams, un vocabulaire de mots-clés et nous utilisons ce vocabulaire lorsque :

" nous souhaitons discuter moult processus centraux à notre vie commune. » $(1976: 12)$

Williams développa par la suite sa liste de motsclés, comme l'ont fait d'autres auteurs tels que Bennett, Grossburg et Morris (2005). Les anthropologues ont eux aussi porté attention à des

* Professor of Anthropology, Associate Dean, Henry Kendall College of Arts and Sciences, University of Tulsa USA, lamont-lindstrom@utulsa.edu

Traduit de l'anglais par Alexis Black et Christine Jourdan. Université Concordia. 
mots-clés importants afin de donner un aperçu d'une culture ou d'une autre. Kim (2005) examina quinze mots-clés en coréen (e.g. " pleurs ", « cuillère ", " feu »); Wierzbicka (1997) compara des locuteurs anglophones avec des locuteurs russes, polonais, allemands et japonais qui partagent des mots-clés importants (tels que " amitié ", « liberté ", " patrie ») bien que les connotations locales de ces mots divergent.

Williams soutient que les mots-clés sont signifiants de « façon matérielle et historique » (1976 : 20) ; c'est-à-dire que les mots-clés façonnent les luttes et expériences quotidiennes tout comme ils sont façonnés par celles-ci. Les mots-clés et leurs significations changeantes peuvent révéler ce qui se passe dans les esprits des individus. Mon propre intérêt pour les mots-clés s'est déclenché lors d'entretiens réalisés avec des amis de Tanna, d'anciens villageois dont plusieurs vivent aujourd'hui dans des quartiers informels de Port-Vila (Lindstrom, 2011, 2011a-b) ${ }^{1}$. Lors de ces entretiens, les mêmes mots revenaient continuellement. Lorsqu'ils pensaient à la vie en ville, ces citadins faisaient des comparaisons géographiques entre la vie urbaine et la vie insulaire, c'est-à-dire entre leur cadre de vie actuel et celui de leur vie précédente. Au-delà des questions de village et de ville, un urbanisme émergeant façonne fondamentalement le développement des cultures nationales à Vanuatu et à travers la Mélanésie. L'expérience de la vie en ville, et non plus celle du village idyllique, génère des expériences partagées et une identité nationale façonnant ce que cela veut dire d'être un ni-Vanuatu, un insulaire des îles Salomon ou un citoyen de la Papouasie Nouvelle-Guinée.

Lorsqu'ils racontaient leur expérience de la migration, les gens parsemaient de mots-clés les comparaisons qu'ils faisaient entre la vie au village et la vie en ville. Au fur et à mesure qu'ils cultivaient leurs nouvelles vies urbaines, ils élaboraient des mots et des termes pour en parler. Pour évaluer leur vie en ville, mes amis de Tanna ont, à plusieurs reprises, utilisé un certain nombre de termes qui ont attiré mon attention. Suivant en cela Raymond Williams, je considère ces termes comme des clés de la vie urbaine à Port-Vila qui reflètent les expériences matérielles et historiques des gens. Les mots-clés donnent un aperçu de l'urbanisme émergeant en Mélanésie. Puisque j'ai fait mes entretiens dans la langue nafe (kwamera) du sud-est de Tanna, ces motsclés venaient parfois du nafe, alors que d'autres venaient du bislama (le pidgin anglais qui est la langue véhiculaire de Vanuatu). Parfois, les narrateurs mélangeaient les langues au cours de la conversation, alternant souvent entre un mot en nafe et son équivalent en bislama. J'ai sélectionné ces mots, non pas en fonction de leur simple fréquence quantitative, mais plutôt en utilisant une analyse narrative : un mot pourrait être " clé » en raison de son occurrence, sa prépondérance, le contexte de signification et, également, la prosodie du locuteur.

Les mots-clés urbains notables que j'ai tirés des récits des immigrants comprennent jalus ("jalousie, être jaloux", etet en langue nafe, bien que le mot signifie plus finement "garder jalousement "); noes (" bruit ", taragaru en langue nafe); sekiuriti (" sécurité", en particulier un agent de sécurité); mobael (à la fois le téléphone et les forces militaires du Vanuatu) et fri ( "liberté " et aussi " gratuit ", auar en langue nafe, pour parler de quelque chose qui ne coûte pas d'argent). On pourrait ajouter à cette liste $t a-$ nak, un calque nafe récent de thanks («merci») ou tangkyu en langue bislama. Le mot wantok (" même langue " ou " camarade ") est un autre mot mélanésien typiquement urbain (Levine et Levine, 1979 : 70 ; Schram, 2015), bien qu'il soit plus courant à Port Moresby qu’à Port-Vila. Le mot-clé principal que je considère ici, le mot respek, s'est avéré l'un des termes les plus utiles à mes amis pour évaluer la vie en ville. L'expérience urbaine a fréquemment provoqué des enjeux de respek (ou rispek en bislama; nisiaiien en nafe ${ }^{2}$ ). Et en parlant de respect, les gens ont surtout dit qu'ils aspiraient à recevoir, et même ressentaient le besoin, de plus de marques de respect (Lerche, 2008).

\section{Respek}

Je laisse aux autres la tâche de reconstituer une généalogie du concept de respect, mais mon hypothèse est que l'exacerbation des manœuvres politiques basées sur l'ethnicité et sur le genre presque partout a sensiblement amplifié l'utilité de ce terme, y compris dans les environnements sociaux complexes des villes postcoloniales mélanésiennes. Williams et ses successeurs n'ont pas inclus le mot " respect " dans leurs inventaires de mots-clés contemporains, mais les futurs chercheurs pourraient bien le faire. En effet, Ngram Viewer de Google indiquent que les usages littéraires de l'expression "demander du respect » ont presque doublé entre 1960 et 2008 . Au cours du

1. Je remercie la Fondation Wenner-Gren de la Recherche Anthropologique et le Pacific Alternatives Project du département d'Anthropologie sociale à l'Université de Bergen de leur soutien de ma recherche sur le terrain au Vanuatu en 2010 et 2011. Je suis aussi redevable à Joel Iau, Harry Iapwatu, et d'autres bons samaritains de leur amitié et de leur conversation pratique, comme toujours.

2. Ceci devrait être écrit nisiaiien où [i] est la voyelle centrale; pour faciliter la transcription, je remplace cette voyelle avec d'autre (semblables) graphiques de voyelles. 
$\mathrm{Xx}^{\mathrm{e}}$ siècle, la montée des discours sur les droits civiques, le féminisme (condensé dans la chanson à succès d'Aretha Franklin "R-E-S-P-E-C-T»), le multiculturalisme, la politique identitaire, ainsi que la mondialisation, la migration urbaine, le choc des civilisations, les conflits religieux - tout ceci a encouragé bon nombre d'entre nous à exiger d'être respectés, ou à se plaindre de ne pas l'être. Les ethnographes des quartiers déshérités du centre de certaines villes ont documenté la présence de demande de respect de plus en plus fortes (par exemple, In Search of Respect de Philippe Bourgois [2003]), tandis que des sociologues ont constaté la même chose dans leurs études des interrelations modernes difficiles. Par exemple, Sara Lawrence-Lightfoot a observé que :

" chaque matin le journal contient une histoire qui fait écho aux paroles de l'hymne d'Aretha: appels en faveur du respect des personnes qui ont été violées, négligées, ou ignorées par leurs proches, par des connaissances, des étrangers, ou des personnalités publiques. » (1999: 8)

La formule accrocheuse de l'acteur comique Rodney Dangerfield, "I don't get no respect " (Je n'obtiens pas le respect qui m'est dû), fait encore écho aux sentiments de beaucoup de gens.

En constante expansion, les centres urbains en Mélanésie sont multiethniques, multilingues et stratifiés économiquement ; ils représentent des microcosmes de respect. Les nombreuses forces qui, ailleurs, poussent les gens à se préoccuper de respect, bouillonnent ici aussi. La population de Port-Vila est en pleine augmentation, passant de 29000 habitants en 1999 à plus de 50000 habitants aujourd'hui. Les dictionnaires et manuels de bislama suggèrent que la préoccupation des gens pour le respect ("respect-worry») a augmenté au fur et à mesure du développement de l'urbanisation qui, au Vanuatu, a pris de l'ampleur de façon importante immédiatement avant et après l'indépendance du pays en 1980 (Haberkorn, 1989 : 9-14). Le mot respek apparait dans le dictionnaire de bislama écrit par T. Crowley en 1990, mais il est absent du manuel de bislama écrit par J. Guy en 1974.

Les migrants urbains originaires de Tanna ont adopté le mot respek avec enthousiasme. Ils en déplorent l'absence et pensent que cette absence explique les conflits et les déceptions auxquelles ils font face. En portant attention à la façon dont les immigrants parlent du respect, on peut comprendre la source de leurs doléances. En Occident, la notion de respect est inhérente à la façon dont on considère les gens (on pense aux marchands de crack du Barrio (Bourgois, 2003 : 324), en quête de « dignité et d'épanouissement ») et aux marques de respect à l'égard de l'autorité, de la tradition, ou des institutions. Au Vanuatu, les bonnes, par exemple, disent qu'elles désirent que leurs employeurs blancs les respectent, sans demander pour autant à être traitées d'égal à égal :

«L'enjeu central pour les bonnes, dès lors, n'est pas pas celui de l'égalité/inégalité, ni de la "différence" "; c'est plutôt un enjeu de respect. Ce que veulent les bonnes et ce dont les bonnes ont besoin, c'est que leurs patrons respectent leurs sentiments et leur dignité en tant qu'êtres humains. " (Kraemer et Rodman, $2005: 148$ )

Le discours de respek au Vanuatu évoque fréquemment des rapports convenables, même s'ils sont généralement hiérarchiques, plutôt qu'il ne revendique l'égalité individuelle.

La question de la personne divisible en Mélanésie est un sujet apprécié, ou controversé, qui a fait couler beaucoup d'encre (Strathern, 1988 : 34). Les différentes conceptions de la personne colorent forcément la compréhension locale des stratégies discursives liées au respect et à ce que les mots veulent dire. Depuis maintenant plusieurs décennies, les ethnographes guettent une montée de l'individualisme possessif en Mélanésie, particulièrement dans des zones urbaines où les immigrants se coupent plus facilement de leurs réseaux familiaux villageois (par exemple, Gewertz et Errington, 1999: 42). Étant donné les frontières poreuses qui existent entre le village et la ville en Mélanésie, on ne peut dire clairement jusqu'à quel point certains migrants urbains ont renforcé leur individualisme. $\mathrm{Ni}$ s'ils insistent davantage qu'avant sur les questions de dignité et de statut personnel. On ne peut dire non plus jusqu'à quel point ils ont atténué les liens avec leur famille et leur village natal. Par conséquent, pour trouver des indices supplémentaires sur la transformation de l'identité individuelle urbaine, on gagnerait à écouter soigneusement les discours que les migrants tiennent sur le respect.

\section{La notion de respect dans les quartiers à habitat informel}

Les quartiers résidentiels urbains sont à la fois semblables et différents des villages insulaires. Les migrants originaires de Tanna qui habitent à Blacksands, Ohlen et Erakor Hafbrij et autres quartiers informels de Port-Vila vivent en général en groupes de familles étendues, certains membres de la famille circulant entre l'île et la ville. Ceux qui s'installent en ville apportent avec eux leurs conceptions de ce qu'est une bonne communauté et essaient, de diverses manières, de façonner les quartiers informels selon le modèle des villages insulaires. Autant que faire se peut, ils en aménagent la topographie, ils plantent des potagers urbains, donnent des noms d'îles aux 
différents endroits de la ville et ainsi de suite (Lindstrom, 2011).

Malgré cela, la vie en ville est particulièrement difficile. Des gens venant de tout le pays habitent en forte proximité dans des regroupements hétérogènes enchevêtrés les uns aux autres. Il se peut que les résidents de ces grappes d'habitats contigus soient aussi des immigrants originaires de Tanna, mais il se peut aussi qu'ils viennent d'ailleurs : il manque généralement à cet assortiment de voisins le soutien familial qui facilite la vie au village (Lindstrom, 2011a-b : 20). Hormis ceux qui ont acheté des parcelles constructibles à Freswota, où elles sont plus petites que dans d'autres quartiers et donc où plus de gens s'agglutinent dans moins d'espace, la plupart de mes amis de Tanna

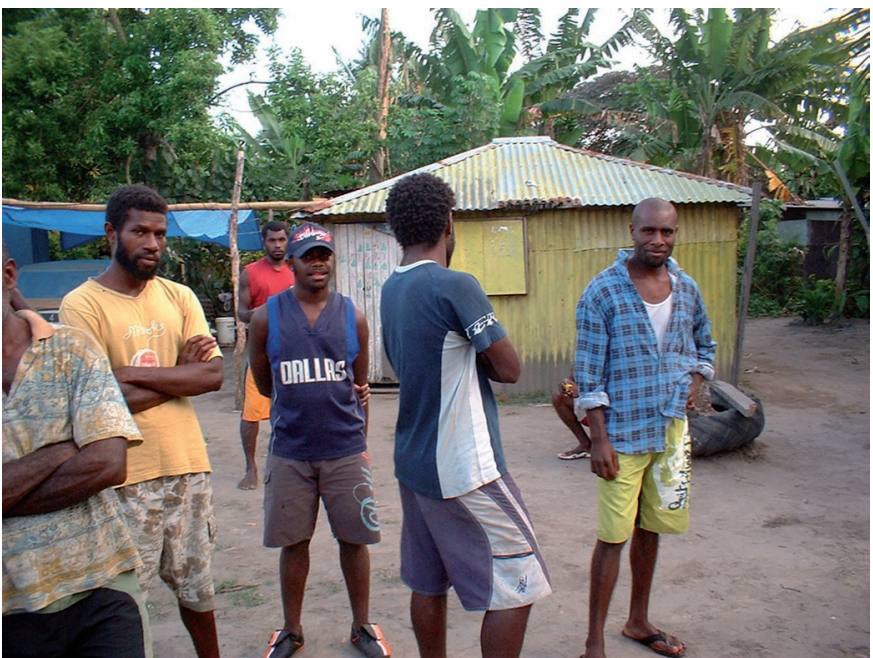

Figure 1. - Immigrants originaires de Tanna dans le quartier de Blacksands, Port Vila, 2010 (C) Lamont Lindstrom) n’ont pas de titre de propriété pour le terrain sur lequel ils ont construit des cabanes en bois et en tôles. Matu, qui réside à Freswota depuis environ quinze ans, a personnalisé sa parcelle en lui donnent le nom de son fils "Ainsle "; mais elle déplore que la vie à Freswota ressemble à une peine de kalabus (" prison»), tellement son espace vital est restreint et ses voisins envahissants.

Vivre dans les quartiers informels peut être éprouvant. Éloignés du village comme ils le sont, les immigrés sont préoccupés par le respect. Si seulement les marques de respect étaient plus nombreuses, la vie en ville serait plus facile. Par exemple, le mot bislama noes («bruit»), en langue de Tanna taragaru, un mot-clé tiré des entretiens, exprimait le malaise que les gens ressentaient devant le désordre et le chaos urbains. L'émergence relativement récente du mot tanak, "merci ", suggère aussi qu'une étiquette urbaine se développe. En l'utilisant, on met en valeur le côté malaisé des relations de voisinage et on urbanise les schémas de réciprocité prévalant dans les îles. Mary Matheson, épouse de missionnaire qui vivait dans l'île de Tanna en 1860, nota que ses voisins insulaires :

« ne semblent pas avoir de mot pour parler de compassion, et on dirait qu'ils ne connaissent pas cette émotion. Il n'y a pas de mot pour exprimer la gratitude. " (Patterson, 1864 : 466)

En ce qui concerne l'expression de la compassion, Matheson avait tort ; mais elle avait raison en ce qui concerne la gratitude car la réciprocité escomptée et obligatoire rend superflue toute expression verbale de gratitude. Le néologisme tanak ajoute un niveau de réciprocité verbale qui apaise la tension souvent présente dans les relations urbaines.

Des conflits, parfois graves, surviennent également dans les villages, mais les résidents ont à leur disposition des schémas et des stratégies fa-

milières pour les régler. En ville, alors qu'ils vivent au milieu de voisins qui ne leur sont pas apparentés, les citadins sont hautement méfiants des motivations des uns et des autres. Ils s'inquiètent, en particulier, de nouvelles sortes de convoitise et de jalousie, jalus en bislama, qui suscitent des ragots, des conflits et même de la sorcellerie (nakaemas). À Luganville, la deuxième ville en importance du Vanuatu, Taylor a noté « un retour troublant au désordre urbain » provoqué par «la mixité ethnique urbaine, les conditions économiques de plus en plus difficiles et compétitives, et le climat de jalus (jalousie) " qui en résulte (2015: 39). Les migrants soupçonnent souvent un acte de sorcellerie jalus d'être la cause des maladies mystérieuses ou des malchances insolites qui ont lieu communément dans les quartiers informels rudes de Vila. L'inquiétude des migrants bouillonne face à ces courants de sorcellerie présents sous la surface des relations sociales (Rio, 2010, 2011). L'angoisse sous-jacente se transforme de temps en temps en confrontations violentes (Mitchell, 2000, 2011).

Préoccupés par la sorcellerie et les conflits actuels et potentiels, les migrants urbains ont vite adopté des notions de sekiuriti - élargissant le sens original du mot anglais pour englober les gardiens et les vigiles qui surveillent les boutiques, boîtes de nuit et distributeurs d'espèces, mais aussi des notions plus générales comme la gestion du tumulte potentiellement dangereux des quartiers informels. À Luganville, par exemple, on dit que des migrants urbains :

« recrutent et s'entourent de sekiuriti (sécurité) humaine, terme qui inclut non seulement les agents de sécurité, mais également les kleva [intermédiaires spirituels] et autres personnes spirituellement puissantes, pour se protéger des possibles attaques de sorcellerie, 
qu'en vertu d'une logique relationnelle, leur positions attirent intrinsèquement. » (Taylor, $2015: 47$ )

En plus de renforcer la sekiuriti, les migrants parlent constamment de respek, exigeant que les relations soient respectueuses, déplorant l'absence de respect, tout en espérant que ce discours améliorera leurs propres chances de survie en ville. Comme dans le cas du mot sekiuriti, la sémantique du mot respek est également multicouche. Sur Vanua Lava, par exemple :

"Si on fait preuve de manque de respect, plusieurs choses peuvent se produire [...] la personne irrespectueuse risque d'avoir un accident. » (Hess, 2009 : 29-30)

Sekiuriti et respek sont tous les deux contrôlés de façon physique et de façon immatérielle. Le manque de respect (tout comme la jalousie brutale) peut avoir un effet boomerang sur la personne irrespectueuse.

Même si Bolton (2003: 4) a trouvé au moins une occurrence du mot respect dans chacune des langues vernaculaires du Vanuatu (voir Vanuatu Cultural Centre, 1999 ; Lerche, 2008 : 5), les gens changent de code (code-shift) librement entre le mot bislama respek et les termes indigènes correspondants. Par exemple, lorsqu'ils parlent de respect dans la langue nafe, les migrants du sud-est de Tanna emploient le mot nisiaiien (le nom), ou siai (le verbe). Aujourd'hui du moins, ce mot se rapproche du sens du mot respect, comme dans l'expression sasiai nekava,

«nous respectons le kava [en étant soucieux des protocoles d'usage]. »

Mais il peut aussi signifier " échec » ou " incapacité » comme dans la phrase :

«le camion risiai la colline " (le camion ne pouvait pas monter la colline).

Les racines sémantiques de nisiaiien ont été vraisemblablement influencées par 150 ans d'usage chrétien. Dans la traduction quil a faite au $\mathrm{XIX}^{\mathrm{e}}$ siècle du cinquième commandement, William Watt, missionnaire et traducteur de la Bible, a employé ce mot dans le sens d' "honneur" :

Tikisiai remam rihnam, ma napen seim rebuk ia tuprana Iehova Atua seim raveipehe mik [" Honore ton père et ta mère, afin que tes jours se prolongent dans le pays que l'Éternel, ton Dieu, te donne. »] (Anonyme, $1941: 15$ )

Sur les douze exemples dans lesquels le mot " respect " apparait dans le Nouveau Testament de la Bible du roi Jacques, Watt n'en a traduit qu'un seul avec le mot siai : "emyaha hi-epesiai ketir kavegi in ya tenari amasan " [ "Et que vous fassiez une distinction envers celui qui porte les tenues plaisantes »]

ou dans la Bible King James,

"And ye have respect to him that weareth the gay clothing" (Jacques, $2: 3$ )

Le mot respek s'entremêle sémantiquement avec le mot honneur - mais doit-on un tel honneur aux individus ou à l'autorité désignée (ses parents ; cette tenue plaisante) ?

Les migrants de Tanna déplorent l'absence de respect, mais ils l'évoquent constamment pour expliquer les conflits urbains et les expériences décevantes qui marquent leur vie. Étant de bons mélanésiens, leurs inquiétudes se focalisent davantage sur la fragilité de l'ordre social dans les quartiers de la ville que sur des problèmes d'honneur, de dignité, ou d'estime personnelle. Cependant, les disparités économiques et "les différences incommensurables" (Gewertz et Errington, 1999 : 42) qui augmentent, risquent de léser de plus en plus l'individu, peu importe l'interprétation. Par exemple, à Port Moresby, pendant les années 1970, ceux parmi les migrants qui étaient des travailleurs non qualifiés ont trouvé la tradition de l'honneur parmi leurs wantoks, tandis que ceux qui étaient plus éduqués ont plutôt trouvé «le respect dans leurs interactions avec leur wantok et les étrangers" (Levine et Levine, 1979: 91). Chez eux, à Tanna, les gens évoquent et réclament du respect. Le sens de siai en nafe, toutefois, ne recoupe peut-être pas tout à fait celui du terme plus large, sémantiquement parlant, de respek en bislama, dans la mesure où les relations interpersonnelles dans les îles sont plus prévisibles et maîtrisables. Mon hypothèse est que les discours sur le respect et les demandes de respect se sont multipliés dans les quartiers informels de Vila au fur et mesure que les migrants ont fait face d'une part à de nouvelles sortes d'inégalité et d'autre part, à la volatilité des relations interpersonnelles.

On trouve à l'annexe 1, traduits en anglais, des extraits de conversations que j'ai eues avec cinq migrants de Tanna sur le thème du respect. Dans ces entretiens, les migrants lient le respect aux problèmes de la communauté, au leadership, à l'accès et au contrôle des ressources limitées et aux responsabilités familiales. Comparant la ville au village, les migrants se plaignent que les citadins manquent, en général, de respect envers les autres. Soarum d'Ohlen Freswin, par exemple, m'a dit :

«Si les gens ne sont pas respectés, ne sont pas respectés, qui est honoré ? Ou, si vous ne respectez pas les autres, vous blessez les gens, et les gens ne vous respectent pas. Aucun respect, le respect [nisiaiien] a disparu. " 
Le manque de respect explique en gros une gamme de problèmes urbains.

«Aucun respect. Les gens font n'importe quoi, les gens volent, ils s'entretuent, ils fument de la marijuana. Les gens ont des mauvaises manières. » (Soarum)

Soarum s'est en particulier plaint de ceux qui dérobent furtivement de la nourriture et du bois de chauffage dans les potagers urbains. Si on était sur Tanna, île où la notion de respect est importante quoique peut-être idéalisée, n’importe qui ayant besoin de quelque chose demanderait d'abord l'autorisation au propriétaire du terrain. À Vila, cette sorte d'échange respectueux et bienveillant tombe en miettes :

"Les gens vont dans tous les sens. Votre terrain? Je vérifie et un homme est entré sur votre terrain. Certains n'ont pas votre autorisation, certains ne volent pas. J'ai noté ceci. Prendre quelque chose de votre terrain, ou y faire quelque chose, ou déblayer la brousse comme sur Tanna. Une personne dégage un terrain [pour un potager]. De ses propres moyens, il y va tout seul dégager un potager. Faites cela et les soucis surviennent, ils surviennent tout de suite. Cet homme, comme je dis, ne respecte pas, il ne respecte pas la propriété d'autrui ni ses biens. Il ne vient pas demander l'autorisation, mais il va directement chercher des ennuis. S'il fait ça et si vous le défiez, vous vous battrez vous deux, et vous vivrez dans les ennuis. »

D'autres, désespérés par les ordures urbaines de Port-Vila, gribouillent " respect " sur les plateformes de bord de la route où on dépose les déchets ménagers pour la collecte hebdomadaire.

Le manque de respect déclenche des conflits urbains, même des bagarres ; les stratégies coutumières de résolution des disputes qui prévalent au village sont moins efficaces dans les quartiers informels socialement compliqués de Vila. Un appel à la police ou aux forces paramilitaires mobael peut créer bien plus de problèmes. Mitchell (2011) a décrit le point de vue ambivalent des habitants des quartiers informels sur les interventions des forces mobael. Ils aiment leurs uniformes de couleur vive, les armes et les bottes noires ; mais ils craignent, avec une certaine justification, de se frotter à eux.

En réponse à tout ceci, les migrants ont inventé des stratégies informelles pour moduler et résoudre les conflits urbains. Le taon jif en est l'exemple le plus notable (Lindstrom, 1997 : 226 ; Rousseau, ce numéro, pp. 37-50). Les chefs de ville sont apparus au fur et à mesure du développement des quartiers informels après l'indépendance du Vanuatu en 1980. Les migrants, partageant des liens de parenté et des origines dans les mêmes villages insulaires, ont reconnu de façon informelle un groupe de porte-paroles qui font office de chefs de communauté. Ceux-ci in- terviennent, si nécessaire, et tâchent d'atténuer les conflits au sein des communautés de migrants ou de négocier et résoudre des conflits qui éclatent entre les différentes communautés. Cependant, la médiation par le taon jif ne réussit pas toujours. Et le manque de respect, encore une fois, en est l'explication. Les chefs eux-mêmes ne sont pas respectueux, se plaint Soarum :

" Je dis que le manque de respect cause toutes sortes d'ennuis. Certains sont des hommes importants, mais ils ne respectent pas les autres. "

Et les demandes de respect viennent aussi de l'autre côté puisque les membres de la communauté eux mêmes ne sont pas respectés. Le Bislama Dictionary de Crowley cite ainsi l'usage prototype du mot respek:

"Ol yangfala long taon samtaem oli no gat respek blong ol jif. [Les jeunes gens en ville parfois manquent de respect envers les chefs]. » (1990: 197)

Même si les taon jifs interviennent, les conflits peuvent ne pas être résolus. Un soir de 2014, après le kava, je rentrais à la maison en traversant Ohlen avec deux jeunes hommes chargés de me protéger - leurs parents étaient soucieux des risques d'une balade nocturne dans le quartier. Bafouillant et criant de façon incohérente (c'est mon impression), un agresseur furieux est survenu derrière nous et a frappé avec force un des garçons au visage, lui ouvrant la joue. C'était son voisin, aussi de Tanna, mais du secteur de White Sands; il était fâché parce que le fils de mon ami avait cassé une assiette sur laquelle l'assaillant s'était accidentellement coupé le pied - d'après ce que m'ont dit les gens qui m'en ont parlé. Les deux familles impliquées ont appelé leurs chefs de village et ont convoqué des voisins à trois réunions de règlement de conflit, tout cela en vain car le parti de l'assaillant a refusé de participer à ces réunions.

De nombreuses discussions sur le respect concernent les vêtements des citadines. Ces dernières années, un débat continu portant sur le troasis (pantalon) - est-il convenable pour les jeunes femmes de porter des pantalons? - a connu des hauts et des bas à Port-Vila (Cummings, 2013). Mon impression est que les femmes en ville ont gagné la guerre des pantalons, mais les migrants continuent de rouspéter en disant que les vêtements féminins doivent envoyer des messages respectueux. Glenda, par exemple, s'est plainte :

«La vie est devenue très difficile. Avant, le respect existait. Avant, quand vous alliez en ville et vous voyiez une femme, vous saviez que c'était une femme ; vous alliez en ville et vous voyiez un homme vous saviez que c'était un homme. Mais aujourd'hui, c'est comme dans cette chanson de $\mathrm{Naio}^{3}$, "Babylonian Style". Vous

3. Naio est un chanteur célèbre dans un groupe de musique de Tanna. 
voyez une femme et vous ne savez pas si c'est une femme. Elle se tourne et elle vous regarde et alors vous notez ses seins. Et toute sorte d'habillement. "

\section{Soarum s'est fait l'écho de la plainte de Glenda :}

«Les femmes portent des pantalons, elles n'ont aucun respect pour les autres aujourd'hui. La vie est devenue mauvaise. La vie est devenue mauvaise, pas bonne. »

\section{Idem pour Iapwatu :}

"Aucun respect, c'est sérieux, les vêtements ne sont pas bien. Aucun respect ici à Vila.»

Il est révélateur que le site de wikivoyage.org fournissant des conseils aux touristes qui vont au Vanuatu associe le respect à une tenue de bon goût. Sous la sous-rubrique "Respect ", le site avertit la touriste prudente de faire attention à sa tenue :

«Dans l'ensemble du Vanuatu, et particulièrement en dehors de Port-Vila dans les villages, la vie est fortement influencée par la "kastom" - un ensemble de coutumes et de tabous traditionnels qui s'appliquent à toutes sortes de sujets. Faites attention à cela, et respectez les demandes des villageois en ce qui concerne la "kastom". Lors de leur visite dans les villages, les femmes devraient s'habiller de façon modeste, porter des vêtements qui couvrent les épaules et également les genoux... Il est déconseillé de porter des vêtements suggestifs ou sexy (comme le fait de porter des vêtements de plage dans la capitale) car un siècle de travail missionnaire a influencé la perception de ce qui est considéré comme un vêtement respectable dans les îles. Dans tous les cas, ce genre de tenue est vue comme un manque de respect aux habitants locaux et peut être interprété par des habitants indigènes comme une invitation aux relations sexuelles. " (wikivoyage.org, $2016: \mathrm{np}$ )

Je pense que ces doléances sur les vêtements ont un fondement plus important : une inquiétude à propos de la responsabilité familiale (et, oui, cela inclut le sexe) dans les nouveaux contextes sociaux que sont les quartiers informels, où chacun essaye si possible de gagner sa vie en travaillant. Les épouses, ainsi, gagnent parfois plus que leurs maris sous-employés. On laisse libre cours aux enfants et Dieu seul sait ce que font les filles ! Elles paradent dans la ville en pantalon ou encore pire. Les pères se lancent dans des batailles perdues d'avance pour maintenir la coutume du mariage telle qu'elle se pratique à Tanna (arrangé par les parents et basé sur l'échange de soeurs entre familles). Le fait de se soucier du caractère respectueux des vêtements reflète d'autres soucis plus profonds sur la dynamique familiale en ville. D'une part des inquiétudes au sujet de fri - le côté négatif de la liberté - qui pousse les gens à négliger leurs devoirs familiaux. D'autre part, l'anxiété dominante provoquée par le téléphone mobael ou portable, omniprésent, qui permet lui aussi l'indépendance personnelle autant qu'il facilite le contact interpersonnel (Anderson, 2013; Lipset, ce numéro, pp. 195-208). Encore une fois, la plupart de ces inquiétudes concernent les jeunes femmes qui se servent de téléphones mobael pour appeler leurs petits amis ou prétendants, esquivant ainsi le contrôle parental (Kraemer, 2015 ; Taylor, 2016).

Bien sûr, l'opinion des migrants urbains sur la liberté (fri) est ambiguë. Soarum, par exemple, se souvient qu'il avait l'impression d'être un " esclave " lorsqu'il travaillait pour UNELCO, la compagnie d'électricité de Port-Vila. Il n'avait aucun contrôle sur ses heures de travail et il devait se soumettre à un patron tatillon. À la recherche de plus de liberté, plus de fri, il a quitté ce travail et a acheté un taxi. Cette sorte de fri est une bonne chose. D'un autre côté, la liberté urbaine peut permettre à des migrants de se soustraire aux responsabilités et aux devoirs envers leurs familles et leurs voisins, particulièrement quand ceuxci viennent demander de l'argent. Les enfants, aussi, tirent profit des occasions qui s'offrent à eux en ville pour échapper au contrôle parental. Ils flânent; ils s'habillent de façon flamboyante et tordent leurs cheveux dans des styles criards; ils chuchotent dans leurs téléphones en quête de romance; ils n'écoutent pas. Ainsi, ce fri en progression est-il une des causes profondes de l'impression d'irrespect qui augmente en ville, et en conséquence, de l'intensification des appels au rétablissement des relations respectueuses.

Dans les quartiers informels de Vila, les migrants trouvent la liberté et font face au manque de respect, mais en fait, comme l'a fait remarquer Iapwatu, de nombreuses turpitudes modernes ont pris naissance à l'étranger :

"Il n'y a pas de respect ici parce que les gens vivent selon la vie de la ville, copiant le mode de vie occidental des hommes blancs : il est arrivé ici et a causé la disparition du respect. »

Glenda, elle aussi, a trouvé que le manque de respect avait des origines étrangères :

"Les gens essaient d'adopter un mode de vie, ils adoptent un mode de vie qui vient d'outre mer. Je dis que c'est comme ça que vivent les étrangers. »

Mais le manque de respect propre à la ville s'étendra-t-il des quartiers informels jusqu'aux villages harmonieux de Tanna ? Uri, qui a habité à Vila très longtemps, a parlé de Tanna comme d'un modèle de paix et de tranquillité :

"Quand nous sommes à Tanna, tout le monde nous respecte et nous avons tous beaucoup d'amour. On trouve paix [et tranquillité] sur Tanna.» 
De son côté, Iapwatu a attribué la présence durable du respect dans les îles à l'influence de l'église et de la kastom:

"Sur Tanna, je pense que c'est peut être la kastom et l'église qui instaurent le respect. "

Cependant, Soarum a des doutes. Il craint fort que le manque de respect se soit déjà propagé de la ville au village :

"C'est comme ça ici, vraiment comme ça à Vila, mais également sur Tanna, c'est pareil, la même chose. C'est pareil dans d'autres pays, et au Vanuatu aussi. Les gens ont adopté ces manières ; ils ont adopté la façon de la ville et l'ont rapportée dans l'île ; c'est pareil. Nous avons adopté cette vie aujourd'hui, une mode, une seule mode. Quelque soit la manière dont on vit ici en ville, c'est la même chose sur l'île. "

Ainsi, la généalogie du manque de respect commence à l'étranger, envahit la ville de Port-Vila, et corrompt maintenant même les foyers insulaires où le respect devient une préoccupation locale, pendant que de nouvelles sortes d'inégalités économiques et disproportionnées se propagent de la ville jusqu'à l'île. Dans les quartiers informels de Vila, et maintenant même dans les foyers villageois, le discours sur le respect transforme et influence une culture nationale émergente. Même si le manque de respect est important, depuis 1980, le respek est devenu une valeur nationale partagée et une stratégie utile pour créer une communauté plus élargie.

\section{Le respect et la culture nationale}

Surtout parce qu'il est souvent absent, le respek est une composante éthique centrale de la culture urbaine ni-Vanuatu, laquelle devient de plus en plus la culture nationale du Vanuatu. Le discours sur le respect envahit les discours urbains et nationaux et ce, au-delà des quartiers informels. Il orne la constitution du Vanuatu dans son chapitre intitulé "Droits fondamentaux et libertés de l'individu ", dont l'un des devoirs fondamentaux est :

« de respecter les droits et les libertés des autres et de coopérer entièrement avec les autres dans l'intérêt de l'interdépendance et de la solidarité. » (7(f))

En outre, un enfant est sensé :

$$
\text { « respecter ses parents. » (7(i)) }
$$

Les agences du secteur judiciaire du pays, y compris le Département du service correctionnel, revendiquent la devise

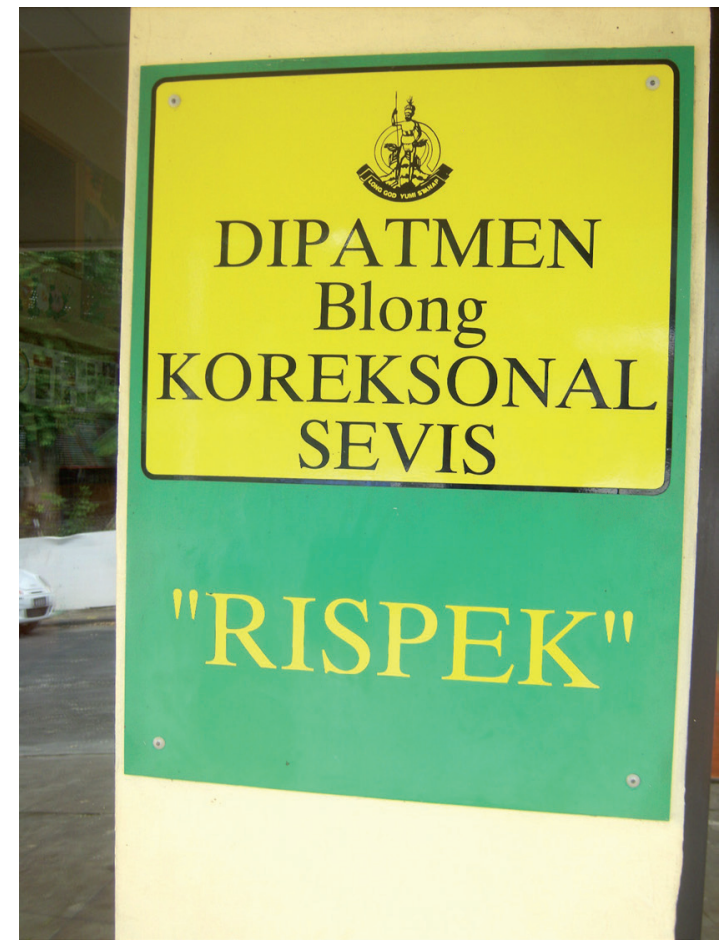

FIgURE 2. - Respect nationaliste sur la porte d'entrée du Département du service correctionnel, PortVila, 2016 (C Lamont Lindstrom)

"Sefti, digniti, rispek mo gud fasin blong evriwan [Sécurité, dignité, respect et bonnes manières pour tous]. »

Bien qu'il soit mort depuis 1999, on salue encore aujourd'hui le premier Premier ministre du Vanuatu, Walter Lini pour sa devise souvent répétée "Le respect est honorable. " Cette phrase a été utilisée depuis dans des contextes inattendus, y compris sur la page Facebook du Bureau de tourisme du Vanuatu, dans un discours de l'ambassadeur chinois, lors des fêtes de l'école secondaire de Malapoa, dans des rapports de consultation sur le développement, et même dans une annonce Airbnb à Port-Vila. L'expression est tirée de la conclusion que Lini a écrite dans un livre publié en 1980 et qui célébrait l'indépendance récente du Vanuatu. Considérant l'avenir du nouveau pays, Lini écrivit :

"Nous croyons que ce qui est petit est beau, que la paix est une force, que le respect est honorable, et que la communauté est à la fois judicieuse et pratique pour le peuple de Vanuatu. » $(1980: 290)$

Rares sont ceux qui saluent aussi l'importance qu'il attribue à la petite dimension du pays, à la paix, à la sagesse et aux dimensions pratiques de la communauté. C'est plutôt le respect honorable qui inspire la mémoire nationaliste.

Le discours sur le respect occupe l'espace public et ce, au-delà du discours officiel. Entre avril 
2014 et mai 2015, on relève dans les archives numériques du journal Daily Post plus de cinq cents occurrences du mot "respect " (écrit respek ou rispek), même si certaines de ces occurences sont des prépositions comme "en ce qui concerne" [in respect of] ou "par rapport à " [with respect to]. Le mot respect apparaît à la Une des journaux, comme par exemple dans un rapport sur le discours d'ouverture du parlement prononcé par l'ancien président Iolu Abbil :

«Président : Enseignez à la jeunesse la valeur du respect et de la fidélité. » (30 mai 2014)

\section{Le Président Abbil a rappelé aux députés et à la nation}

"l'importance d'inculquer à la jeunesse les valeurs de fidélité et de respect."

Le respect revient fréquemment dans des reportages et des commentaires sur l'actualité. Par exemple, la responsable de la biodiversité du Vanuatu, une femme que les gens estiment, prône le respect pour les roussettes et les oiseaux :

"La très respectée directrice de la biodiversité a dit : "Il est important que les gens sachent que les habitats et l'alimentation de cette faune sont également affectés comme nous les humains, donc les gens doivent respecter ces animaux et ne pas les chasser.” " (23 mars 2015)

Le Daily Post rapporte souvent que les politiciens et les chefs demandent qu'on les respecte, faisant écho à l'usage prototypique du dictionnaire de bislama (Crowley, 1995). L'ancien premier ministre Natuman, originaire de l'ouest de Tanna, a compté jusqu'a cinq « principes ou valeurs de kastom" qui serviraient d'assise à son gouvernement, "les N-règles d'or " (beaucoup de noms dans des langues de Tanna commencent par « $\mathrm{n} »)$. La deuxième de ces règles d'or est, en effet, nisiaiien

"qui veut dire "respect". Ce principe a été fortement recommandé par les pères fondateurs du Vanuatu, dont Fr. Walter Lini qui a dit, "le respect est honorable". Si nous nous respectons nous-mêmes, nos voisins et nos communautés, on n'aura pas besoin de gaspiller les ressources précieuses et limitées en surveillance policière dans nos centres urbains » $\left(14\right.$ juin 2014) ${ }^{4}$

En mars 2016, un deuxième premier ministre a de même exhorté " les citoyens du Vanuatu à continuer à soutenir et respecter le travail des chefs dans toutes les communautés du pays," après qu'il ait lui-même reçu en cadeau, comme "signe coutumier de respect", une natte, un cochon et du kava (Ligo, 2016).
À l'instar des présidents et premiers ministres, d'autres dirigeants et chefs font eux aussi régulièrement la promotion du respect - surtout le respect pour la kastom:

"Le Chef Worwor fit la déclaration suivante : "Organisez des réunions de jeunes garçons et de jeunes filles et apprenez leur les valeurs traditionnelles telles que le respect, les allocutions publiques, la cuisine traditionnelle, le tissage des nattes et de paniers coutumiers, les danses traditionnelles, les chants, les mythes et les légendes, l'histoire de leurs îles, l'apprentissage du leadership traditionnel, et encore davantage". " (5 mars 2015)

De la même façon, le chef Abert Moses Mariasi fit l'éloge de quatre-vingts-quatre nouvelles résolutions que d'autres chefs de Tongoa et lui même avaient rédigées. Il a proposé que ces résolutions constituent :

" un guide qui aidera également notre jeune génération de plusieurs façons, comme le fait d'apprécier notre mode de vie culturel, de se respecter les uns les autres, de respecter notre île et notre peuple, d'apprendre les modes de vie traditionnels et même le leadership, l'unité, la paix, le processus de réconciliation traditionnel, le mariage coutumier et tout le mode de vie traditionnel ainsi que toutes ses lois coutumières et même plus. » (6 avril 2014)

Les lettres à l'éditeur évoquent constamment le besoin de respect. Ainsi, man-kam (bislama pour " migrant »), un "Residen' de Port-Vila», a écrit au journal pour demander qu'on respecte les sites culturels locaux situés près de Freswota:

Yumi ol man-kam yumi mekem inaf damej finis long ol kaljoral site blong ol man ples. Yumi respektem olgeta from yumi stap long nasara blong olgeta. No go tumas long stael blong whiteman from stael ya hemi blong self destruct nomo. [Nous les migrants avons déjà endommagé les sites culturels des personnes d'ici. Nous les respectons [les habitants locaux] car nous vivons dans leur nasara [le terrain de danse du village]. Ne suivez pas excessivement le style de l'homme blanc, parce que ce style est simplement suicidaire.] (31 janvier 2015)

Une autre personne écrivit au journal pour protester contre le viol, exigeant qu'on respectât les femmes et les filles :

«Be igud blong ol lida long komuniti i toktok long ol yangfala mo ol olfala blong oli rispektem ol woman mo gel " [Toutefois, c'est bien que les chefs de la communauté apprennent à la jeunesse et aux aînés à respecter les femmes et les filles] (15 avril 2014)

Je pourrais continuer à énumérer les exemples. Le discours sur le respect se manifeste dans

4. Les autres, selon le rapport, sont nolkeikeian (" amour»), nasituan (" aide, générosité »), nuparhianan ("vérité, honnêteté ») et nawitan ("bonne volonté, vertu»). 
d'autres médias que les journaux. Des amateurs ni-Vanuatu de courrier électronique célèbrent le respect en l'utilisant dans les formules de politesse qui terminent leurs messages. C'est ce qu'a fait Anderson Ismael le 5 février 2015:

\author{
«Bonjour frère, \\ Merci. \\ Passe une journée bienheureuse aujourd'hui \\ Salut... \\ Rispek»
}

Les membres de groupes Facebook du Vanuatu, tel que Yumi Toktok Stret ("Nous parlons franchement »), évoquent également souvent le besoin de plus de respect/respek ou en déplorent l'absence. Des messages quotidiens de ce groupe en sont la preuve. De son côté, Abel Nako, un membre de la petite communauté littéraire du Vanuatu, a co-écrit un roman sentimental sur le respect: Vanuatu: Un conte de respect et d'unité (Jensen et Nako, 2014). Dans ce roman, la sagesse du chef et les valeurs de kastom inculquent à la jeunesse d'Ambae :

«le respect pour leurs parents et un apprentissage des valeurs établies " (Daily Post, 5 mars 2015)

en dépit des expériences aliénantes vécues au pensionnat.

Les ONG, dont les sièges sociaux se trouvent à Port-Vila, cultivent aussi un discours de respect propre au Vanuatu. Ces groupes, qui veulent promouvoir l'égalité entre les sexes et qui se battent contre la violence domestique, ont embrassé le discours sur le respect afin de plaider en faveur du droit de la femme et de la paix des familles (Tor and Toka, 2004 : 14 ; Lerche, 2008 : 1112). En 2014, l'UNiCEF a financé un "Camion du respect " dans le cadre d'un programme scolaire contre l'intimidation. Le Camion du respect s'est déplacé dans toutes les écoles de PortVila et d'Efate. Pour ce programme, l'UNICEF a fait écrire une chanson "Se respecter les uns les autres " par le musicien local Stan Antas. Prêchant le besoin de "Leftem Up Rispek», son premier verset conclut :

"Rappelez-vous ceci : Se mi mi olsem yu, yu yu olsem $m i$ / Il faut qu'on se respecte les uns les autres, se respecte les uns les autres. (https://www.unicef. org/pacificislands/1852_23312.html et https://www. youtube.com/watch? $\mathrm{v}=\mathrm{Bj}$ WW8YsKNHes\&feature $=$ yo utu.be)

Ces traitements nationalistes, journalistiques et organisationnels du respect complètent et renforcent les conversations quotidiennes sur ce sujet parmi les migrants urbains de Vila. Ensemble, ces traitements contribuent à la certitude nationale croissante que le Vanuatu est - ou au moins devrait être - un environnement respectueux.

\section{Respectueusement vôtre?}

Les migrants de Tanna vivant à Vila trouvent habituellement que la vie en ville est difficile, surtout quand ils comparent l'expérience urbaine et la vie dans leurs villages d'origine. Les nombreuses plaintes de Kieri sur ce sujet sont typiques :

" Je vois que la vie à Vila est dure. Dur, si vous voulez vivre, si vous voulez rester, vous devez travailler. À cause du vatu [l'argent]. Et si vous ne travaillez pas, c'est plutôt dur pour vous. Le transport, c'est comme ça, la vie à Vila. Vous voulez dormir, il faut payer. Vous payez une maison, vous payez la lumière, payez l'eau, vous obtenez à manger avec l'argent, si vous allez aux toilettes vous payez!»

Ce qui, par le passé, était fri sur l'île est maintenant commercialisable en ville. À Port-Vila, le discours sur le respek coexiste avec les plaintes habituelles, comme celles de Kieri, à propos d'argent. Il révèle le mal-être existant en ville au sein des familles et entre voisins à cause de l'érosion des systèmes d'échange réciproque et de partage à la demande. Il signale aussi une anxiété liée à la possibilité de conflit entre voisins et à la crainte que des personnes esquivent les responsabilités qui leur incombent sur Tanna. Si tanak asori (" grand merci ») est en effet une invention de la période post-contact, on peut en déduire quavant [le contact avec les Européens], les gens de Tanna se sentaient peut être moins obligés de récompenser quelqu'un qui avait simplement respecté ses obligations d'échange et de don. Et, même aujourd'hui, ceux qui utilisent cette marque de politesse s'échangent simplement un grand tanak (merci), mais ne personnalisent pas le remerciement au moyen de lexèmes appropriés.

Le discours sur le respect s'amplifie en parallèle avec l'individualisme, non parce que les migrants urbains, comme dans les bandes dessinées américaines médiocres, insistent sur la notion de respect, mais parce que les gens désirent encore entretenir des identités communautaires et relationnelles. Les migrants tiennent toujours un discours sur le respect qui vise les responsabilités relationnelles d'une personne, en ville autant qu'au village, même si les difficultés causées par la ville et par les occasions qu'elle offre, érodent ces responsabilités. Dans les quartiers informels de Port-Vila, il n'y a pas encore beaucoup de gens qui, s'identifiant à l'Occident, demanderaient que l'on respecte leurs différences singulières et magnifiques. Nous ne sommes pas encore dans une situation où le mot d'ordre serait "respectueusement vôtre", ou "avec tous mes respects", ou même "avec tous nos respects". Le mot d'ordre urbain est plutôt d'honorer et de respecter le respek lui-même. Le respect sert de marqueur - le canari dans la mine de charbon urbaine - de la force et de la viabilité actuelles des attentes traditionnelles que l'on a des gens et de 
leurs relations; et ces relations qui unissent parents et enfants, maris et épouses, le peuple et les jifs sont intrinsèquement hiérarchiques. Le respek alimente sûrement ces inégalités, ou tente de le faire. Il révèle les idéaux de coopération et d'identité relationnelle qui sont maintenant menacés et corrodés par l'expérience urbaine.

"S'il est absent, on en demande; si on en abuse, on le dénonce. » (Lerche, $2008: 16$ )

Le respek est donc l'antidote, du moins les gens l'espèrent, à l'individualité rampante, à la liberté gênante, et à l'égoïsme. Demander des marques de respect a un effet thérapeutique; c'est un remède potentiel, mais de plus en plus inefficace, pour soulager les conflits existant au sein des familles migrantes, mais aussi entre les différentes familles.

De même, au-delà des quartiers informels, le discours sur le respect suggère la possibilité qu'une communauté nationale existe. Le respect mène à l'unité nationale, au consensus, et à la stabilité. En appeler au respect est utopique, mais c'est également stratégique. C'est ce qu'implique un commentaire écrit sur Facebook le 17 mai 2015 par Robson S. Tigona :

« Respectez tous la loi, que vous soyez policiers ou citoyens blo mekem Vanuatu come wan "golden" [pour transformer le Vanuatu en "un état en or"]. »

Ces guillemets suggèrent que "l'état en or" parfait est un projet ambitieux, mais la manière d'y arriver est claire : soyez respectueux. Récemment, les demandes et les inquiétudes quotidiennes des gens à propos du respek ont servit de base à ce qui pourrait être la quintessence d'une communauté idéale nationale.

Ces demandes grandissantes de marques de respect, de plus en plus bruyantes, devraient cependant préoccuper les migrants urbains de Vila. Plus les gens insistent sur les marques respect, moins ils en trouvent. La culture urbaine émergente sape les conditions coutumières du respek mais pousse les gens à y attacher encore plus d'importance. Pendant que le respek se dissipe, les migrants urbains, comme l'a dit Soarum, "vont dans tous les sens". Tandis que le voyage du village à la ville exige seulement un voyage simple, beaucoup de migrants en ville trouvent la vie dans les quartiers informels difficile et multiforme. Ils continuent d'espérer que le respect rendra leurs voyages harmonieux.

\section{BIBLIOGRAPHIE}

Anderson Barbara, 2013. Tricks, Lies, and Mobile Phones: 'Phone Friend' Stories in Papua New Guinea, Culture, Theory, and Critique 54, pp. 318-334.
Anonymous, 1941. Naresien em Nujpume ia Nafwakien ia Nagkierien Kwamera, in $\mathrm{Nu}$ pume em Napuanmen mine Nabukmin, Kyneton, VICT, Armstrong Brothers.

Bennett Tony, Lawrence Grossberg and Meaghan Morris, 2005. New Keywords: A Revised Vocabulary of culture and Society, Oxford, Blackwell Publishing.

Bolton Lissant, 2003. Unfolding the Moon: Enacting Women's Kastom in Vanuatu, Honolulu, University of Hawai'i Press.

Bourgois Philippe, 2003 (2 $2^{\text {nd }}$ ed.). In Search of Respect: Selling Crack in El Barrio, Cambridge, Cambridge University Press.

Crowley Terry, 1990. An Illustrated BislamaEnglish and English-Bislama Dictionary, Port Vila, Pacific Languages Unit, University of the South Pacific.

Cummings Maggie, 2013. Looking Good: The Cultural Politics of the Island Dress for Young Women in Vanuatu, The Contemporary Pacific 25, pp. 33-65.

Gewertz Deborah B. et Frederick K. Errington, 1999. Emerging Class in Papua New Guinea, Cambridge, Cambridge University Press.

GuY J. B. M., 1974. Handbook of Bichelamarl Manuel de Bichelamar, Canberra, Dept. of Linguistics, Research School of Pacific Studies, Australian National University, Pacific Linguistics C-34.

Haberkorn Gerald, 1989. Port Vila: Transit Station or Final Stop: Recent Developments in Ni-Vanuatu Population Mobility, Canberra, National Centre for Development Studies, Australian National University.

Hess Sabine C., 2009. Person and Place: Ideas, Ideals and the Practice of Sociality on Vanua Lava, Vanuatu, Oxford and New York, Berghahn Books.

Jensen Karin et Abel Nako, 2014. Vanuatu: A Tale of Respect and Unity, Self-published with CreateSpace (an Amazon print house).

KIM Yol-kyu, 2005. Uncovering the Codes: Fifteen Keywords in Korean Culture, Fremont, CA, Jain Publishing Co.

Kraemer Daniela, 2017. 'Do you have a mobile?' Mobile phone practices and the refashioning of social relationships in Port Vila Town, The Australian Journal of Anthropology 28 (1), pp. 39-55 (http://onlinelibrary.wiley. com/doi/10.1111/taja.12165/full).

Kraemer Daniela et Margaret Rodman, 2007. Conclusion, in Margaret Rodman, Danie- 
la Kraemer, Lissant Bolton et Jean Tarisesei (eds), House-Girls Remember: Domestic Workers in Vanuatu, Honolulu, University of Hawai'i Press, pp. 143-151.

Lawrence-Lightfoot Sara, 1999. Respect: An Exploration, New York, Perseus Books.

Lerche Stephanie, 2008. Rispek in Vanuatu: Negotiations of Difference and Equality, Unpublished paper presented at Australian Political Studies Association Conference, Brisbane.

Levine Hal B. et Marlene Wolfzahn Levin, 1979. Urbanization in Papua New Guinea, Cambridge, Cambridge University Press.

Ligo Godwin, 2016 (10 March). PM: Greatest challenge to our culture is external culture, Vanuatu Daily Post.

Lindstrom Lamont, 1997. Chiefs in Vanuatu Today, in Geoffrey White and Lamont Lindstrom (eds), Chiefs Today: Traditional Pacific Leadership and the Postcolonial State, Stanford, Stanford University Press, pp. 211-228.

—, 2011a. Vanuatu Migrant Lives in Village and Town, Ethnology 50, pp. 1-15.

-, 2011b. Urban(e) Tannese: Local Perspectives on Settlement Life in Port Vila, Vanuatu, Journal de la Société des Océanistes 133, pp. 18-29 (https://jso.revues.org/6461).

Lini Walter Hadye, 1980. The Future, in Vanuatu: Twenti Wan Tingting blong Team blong Independens, Suva, University of the South Pacific, Institute of Pacific Studies, pp. 282-291.

Mitchell Jean, 2000. Violence as Continuity: Violence as Rupture, Narratives from an Urban Settlement Vanuatu, in Sinclair Dinnen and Allison Ley (eds), Reflections on Violence in Melanesia, Canberra, Hawkins Press and Asia Pacific Press, pp. 189-208.

-, 2011. 'Operation Restore Public Hope': Youth and the Magic of Modernity in Vanuatu, Oceania 81, pp. 36-50.

Rio Knut, 2010. Handling Sorcery in a State System of Law: Magic, Violence and Kastom in Vanuatu, Oceania 80, pp. 182-197.

-, 2011. Policing the Holy Nation: The State and Righteous Violence in Vanuatu, Oceania 81, pp. 51-71.

Schram Ryan, 2015. Notes on the Sociology of Wantoks in Papua New Guinea, Anthropological Forum 25, pp. 3-20.

Strathern Marilyn, 1988. The Gender of the Gift: Problems with Women and Problems with Society in Melanesia, Berkeley, University of California Press.

TAYLOR John P., 2015. Sorcery and the Moral Economy of Agency: An Ethnographic Account, Oceania 85, pp. 38-50.

_, 2016. Drinking Money and Pulling Women: Mobile Phone Talk, Gender, and Agency in Vanuatu, Anthropological Forum 26, pp. 1-16.

Tor Roselyn et Anthea ToKa, 2004. Gender, Kastom and Domestic Violence: A Research on the Historical Trend, Extent and Impact of Domestic Violence in Vanuatu, Vanuatu Department of Women's Affairs.

Vanuatu Cultural Centre, 1999. Kastom Fasin Blong Respect: 1998 Woksop blong ol Woman Filwoka, 12-24 Oktoba 1998, Port Vila, Vanuatu Cultural Centre.

Williams Raymond, 1967. Keywords: A Vocabulary of Culture and Society, New York, Oxford University Press.

\section{APPENDIX 1: PARTIAL INTERVIEW TRANSCRIPTS}

\section{Harry Iapwatu (Ohlen Nabanga, but often resident in Samaria, Tanna)}

Nisiaiien riuan u iFila. Nisiaiien riuan. Rosi fwe Ipare, kastom mene nafakiien, krauni nisiaiien. Mata ikvehe mara u iFila mata laef sai taon nisiaiien riuan. Nisiaiien riuan, rasori, dresing me napwah namasanien. Mua nisiaiien riuan u iFila. Nisiaiian rouihi iesa te narnah mua hamara ia laef sai taon mamo kopiaot ia Western Style ia naraien sai nipitoga a. Ravehe ia kwopen $i$ na ro pen sai nisiaiien ramiuan. Nisiaiien ramiuan te nepran me neraman me iraham pam.
There is no respect here in Vila. No respect. Perhaps on Tanna, kastom and the church, the two establish respect. But you come and live here in Vila and town life [has] no respect. No respect, it's serious, clothing is not good. No respect here in Vila.

Respect is lacking here because people live according to town life, copying Western style life of the white men. It came to this place and made respect disappear. There is no respect for women or men, or everyone. 


\section{Soarum Isaac (Ohlen Freswin, 1987-1914)}

Mata takwtakwni mesite pehe nah iou iakata nisiaiien riuan Monty. Nisiaien riuan. Nermama iraha hamo apenapen nari, nermama iraha hamakres, nermama iraha hamouasi apune nermama, nermama iraha hamase marijuana. Nermama iraha hamo way me nepwun rapwah namwhenien. Nepran fwe tui iraha hapukamuven mha ia traosis. Mata takwtakwni iesa, iesa iako kamparem 1987 mesite 2014 u, nepran iraha hamuven ia traosis, nepran iraha nisiaiien riuan mwi tenraha takwtakwni. Naraien renameraha. Naraien renameraha rapwah namasanien.

Ro nari ianaha $i$ mua, rosi mua te nepen riti $k a-$ maha tahavehe mho amwhen ia kamiaha, ia kaontri me sakamiaha rosi Amerika ua paku, Australia ua iken Europe ua paku trabol ramo hapen iken, ho mua Vanuatu trame eraha, trapwah namasanien. Mata nisiaiien renamiuan, nisiaiien renamiuian.

Fwe kupwan ramasan, mata takwtakwnu, in i iakuvni raka, nisiaiien Monty, nisiaiien riuan. Nisiaiien riuan. Ierman in ramo pen ia iermama. Ikurkuren a? Na iako ting mua kamaha $i$ ia Vanuatu renamo lusum ia nari nah. Namo lusum ia nisiaiien. Nari riti sai Prime Minister sakamaha Father Walter Lini ramani ua, iahamuvahi independen. Nagkiariien asori iti, nagkiariien savani. In rani ia naha i mua respek, hemi honorable. Ikurkuren. Mata tenua iermama napwah noien respek, nisiaiien rapwah nisiaiien, tro honorem ia si? Ua tenua ikapwah noien respektem ianiram, ikamotukun pen nermama, nermama napwah noien respektem ianram. Respek namiuan, nisiaiien renamiuan.

Na iou iakata naha fwe ia taem naha, ia 1987 iahamuvahi independen ia 1980. Mata 1987, ik ikurkuren, mesite nah takwtakni iakata nisiaiien renamiuan. Neramama iraha hamaven apenapen a. Saim tuprena? Iou iakavehe fwe, iermama iti in raven men fwe saim tuprena. Nepwun hapwah nuvahiien [permission], nepwun hapwah naresien. Mua iou taken pen naha. Muvahi nari riti ia saim tuprena, ua tako nari riti, ua takarai rigi nei me riti, ramwhen ia hamo wok fwe Tanna. In reven apenapen a, marai. Reven atukw a, reven atukw a marai. Ro ia naha i trabol rauta renauta takwtakwnu. Iermama, in a iakuvni, in rapwah nisiaiien, in rapwah nisiaiien neramama savani nah property ua, savani nab narimnari. Trapwah naniien marari, mata in ro a men matui trabol. Tenua ren mo, ik ik tikni, kamirau iranouasi kamirau mi, ua tirauara ia trabol. Nisiaiien riuan. Na iou iakata ia 1987 mesite 2014, nisiaiien riuan. Takani ia naha $i$ mua nisiaiien riuan mata ia trabol mene narimnari me. Nema asori puk anan, mata nisiaiien riuan.

Ramo iesa u, rosi iFila rasori na mata fwe ia Tanna ramo mui a, ramwhen mui a, ramwhen mui. Mata in nah rupukwo mha kaontri apa riti, Vanuatu $i$. Nermama iraha havahi mho adoptem ia way; ua iraha hamuvahi fasin sai taon, mherupen bak fwe aelan, ramwhen mui a. Mene samauta ia naraien naha takwtakwnu, i naraien kwati a, naraien kwati a. Nafe ramara iesa i taon, fwe aelan ramara mui ira.
But now, nowadays, I see that there is no respect, Monty. No respect. People do any kind of thing, people steal, people kill one another, people smoke marijuana. People follow ways that aren't correct. Women in the past didn't go about wearing trousers. But today, here, here I can compare 1987 with 2014 today, women wear trousers, women have no respect for others today. Life has become bad. Life has become bad, not good.

Because of this, perhaps sometime we will become like you all, in your countries like America, or where, Australia, Europe, where much trouble happens, making Vanuatu go bad, not be good. Look, respect has disappeared, respect has disappeared.

Before it was good, but now, as I have said, respect Monty, there is no respect. No respect. A man makes trouble for people. Do you understand? I think that we in Vanuatu have lost that. Have lost respect. One thing our [former] Prime Minister Father Walter Lini said when we won independence. An important speech, his speech. He said that respect is honorable. You understand? But if people have no respect, no respect, who is honored? Or if you don't respect others, you injure people, and people don't respect you. No respect, respect [nisiaiien] has disappeared.

I have seen this from that time, 1978, we won independence in 1980. But 1987, you know, until today I see that respect has disappeared. People go in all directions. Your land? I check there, and a man has gone onto your land. Some don't have your permission, some don't steal. I have noticed this. Taking something from your land, or do something, or clear bush as on Tanna. A person just goes all about and clears land [for a garden]]. On his own, he goes on his own to clear a garden. Do that and trouble will rise, will arise now. That man, as I say, doesn't respect, he doesn't respect a person's property or his things. Doesn't come to ask, but just goes to look for trouble. If he does this, and you challenge him, you two will fight you two, and you will live in trouble. No respect. I've seen this from 1987 to 2014, no respect. I say that no respect leads to trouble with everything. Some are leading men, but they have no respect.

It's like that here, really like this in Vila but also on Tanna, it's the same, the same. It's the same in other countries, and Vanuatu too. People have adopted those ways; they have adopted town fashion, and taken this back to the island, it's the same. We have taken on that life today, one fashion, only one fashion. However one lives here in town, it's the same on the island. 


\section{Uri (Freswota 4, 1992-present)}

Naraien ia Tanna, nisiaiien savahi rasori, nokeikeiien savani rasori. Namarinuien fwe Tanna. Mata sai Fila, nisiaiien rouihi. Nokeikeiian rouihi. Namariunuien rouihi.

\section{Glenda (Ohlen Freswin, 1987-2014)}

Laef ravehe mamo menekeneken puk anan. Rosi kupwan, kupwan iakani a rosi ia nisiaiien a. Kupwan ikavan ia taon mata pran, ikurkuren mua pran; ikavan ia taon mata ierman ikurkuren mua ierman. Mata ipwet mene rambwen ia nupu riti sai Naio ramani "Babylonian Style." Ikata pran ikreirei mua pran. Truvsini a mati pehe ikata nanhen. Kani narkahuien me sapamsapa. Nermama hamo traem te nuvahiien naraien ihi, iraha hamuvahi naraien sa rakw peraha. Iakani ira rosi naraien sai nepitoga.

\section{Kieri Ouihi (Prima, 1980s-present)}

Rosi naraien iFila iakata ianaha i mua rekeneken kwopti. Rekeneken iesa mua, tenua ikara, kani ua tikamara tikakeikei mo wok. Na mata nari vatu. Mata tenua ikapwah noien wok rekeneken ouihi tukw ik. Te navanien, mata in nah, naraien iFila. Ikua tikapri ikapri ikamri a mane. Tikaruku nimwa, ua laet ikaruku laet, maruku nui, rosi navahiien naveganien mane, tiken ia toelet ikaruku!
Living on Tanna, we all have respect, we all have lots of love. There is peace [and quiet] on Tanna. But in Vila, respect is small. Love is small. Peace is small.

Life has become very difficult. Before, before there was respect. Before you went to town and saw a woman, you knew she was a woman; you went to town and saw a man you knew he was a man. But today, it's like that song Naio sang, "Babylonian Style." You see a woman and don't know she's a woman. She turns and looks towards you and then you notice her breasts. And all kinds of clothing. People are trying to adopt a lifestyle, they are adopting a lifestyle than comes from overseas. I say it's like the foreigners live.

I see that life in Vila is tough. Tough, if you want to live, you want to stay you must work. Because of vatu [money]. And if you don't work, it's kind of tough for you. Transportation, it's like that, life in Vila. You want to sleep, you must give money. You pay for a house, you pay for light, pay for water, you get food with money, if you go to the toilet you pay! 